\title{
Lacunarity, predictability and predictive instability of the daily pluviometric regime in the Iberian Peninsula
}

\author{
M. D. Martínez ${ }^{1}$, X. Lana ${ }^{2}$, A. Burgueño ${ }^{2}$, and C. Serra ${ }^{2}$ \\ ${ }^{1}$ Departament de Física Aplicada, Universitat Politècnica de Catalunya, Barcelona, Spain \\ ${ }^{2}$ Departament de Física i Enginyeria Nuclear, Universitat Politècnica de Catalunya, Barcelona, Spain
}

Received: 10 November 2006 - Revised: 23 February 2007 - Accepted: 1 March 2007 - Published: 14 March 2007

\begin{abstract}
The complexity of the daily pluviometric regime of the Iberian Peninsula is analysed from the point of view of its lacunarity, predictability and predictive instability. The database consists of daily pluviometric records obtained from 43 rain gauges in Spain and Portugal for the period 1950-1990. Five different series are generated for every rain gauge. The first series is constituted by the consecutive daily amounts. The other four consist of dry spell lengths with respect to daily amount thresholds of $0.1,1.0,5.0$ and $10.0 \mathrm{~mm} /$ day. A dry spell length is defined as the number of consecutive days with rainfall amounts below one of these thresholds. The empirical lacunarity for every rain gauge is well reproduced by two power laws, the exponents varying notably from one gauge to another. The spatial distribution of the lacunarity is characterised by a north to south or southeast gradient, thus suggesting that this parameter can be a useful tool to distinguish between different pluviometric regimes. The predictability of the five series is quantified by means of the rescaled analysis and the interpretation of the Hurst exponent. Its patterns reveal that most part of the Iberian Peninsula shows signs of persistence for the daily rainfall and the dry spell series, although persistence is only clearly manifested in some small domains. The instability of possible predictive algorithms is analysed through the Lyapunov exponents. They are only computed for the series of daily amounts and for dry lengths respect to the threshold level of $0.1 \mathrm{~mm} /$ day due to the short number of dry spells for larger threshold levels. The series of daily amounts depict the highest instability along the Mediterranean coast. The series of dry spells show an increasing instability from NE to SW Spain, with a relevant nucleus of high Lyapunov values in the south-western Atlantic coast. As a summary, lacunarity and Hurst and Lyapunov exponents depict a relevant spatial variation, which is in agreement with well known patterns of
\end{abstract}

Correspondence to: X. Lana

(francisco.javier.lana@upc.edu) the pluviometric regime, such as annual amount spatial distribution and return periods of dry spells.

\section{Introduction}

\subsection{State of the art}

The fractal nature of the rainfall processes is an accepted behaviour and numerous studies have been published during the last decades. It can be cited Lovejoy and Mandelbrot (1985), Rodríguez-Iturbe et al. (1989), Olsson et al. (1993), Hubert et al. (1993), Tessier et al. (1996), Harris et al. (1996), Veneziano et al. (1996), Svensson et al. (1996), Lima and Grasman (1999), Mazzarella (1999), Mazzarella and Tranfaglia (2000), Sivakumar (2001a, b), Sivakumar et al. (2001) and Salas et al. (2005), among many others. These references include a set of concepts (multifractality, chaotic behaviour, time persistence, predictability) applied to a variety of topics as rain intensity, annual amounts, precipitation caused by convective storms, characterisation and comparison of different climates and design and improvement of rain-gauge networks.

Many features concerning the predictability of a pluviometric regime can be analysed on the basis of the nonlinear behaviour of the time series describing it. It can be cited, among others, the rescaled analysis and the meaning of its Hurst exponent, and also a set of descriptors of the mechanism governing this regime (embedding dimension, correlation dimension, Kolmogorov entropy and Lyapunov exponents) which are based on the concept of strange attractors and on the reconstruction theorem. The present analysis of the pluviometric regime of the Iberian Peninsula is aimed to characterise the time irregularity (lacunarity) of the pluviometric records and to evaluate the success of possible predictive algorithms by means of the interpretation of the Hurst exponent and their instability manifested by the Lyapunov

Published by Copernicus GmbH on behalf of the European Geosciences Union and the American Geophysical Union. 


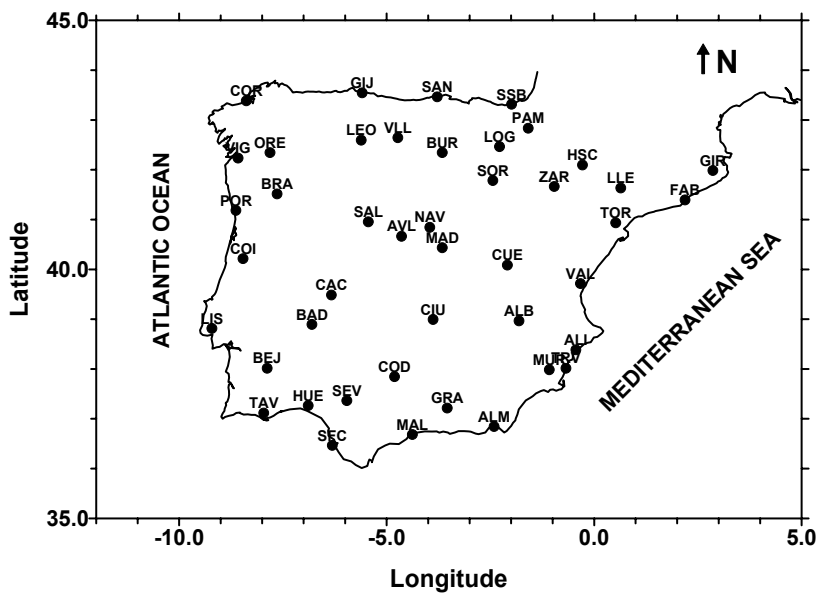

Fig. 1. Geographical location of the 43 rain gauges belonging to the National Meteorological Offices of the Spanish and Portuguese Governments.

exponents. A future deeper characterisation of the nonlinear mechanism governing the pluviometric regime could be achieved by means of the additional concepts linked to the reconstruction theorem.

Database and methodology are introduced in Sects. 1.2 and 1.3. Concepts of lacunarity and predictability and the results obtained for the daily pluviometric regime are detailed in Sects. 2 and 3, respectively. Section 4 is devoted to develop the concept of predictive instability, and includes the mathematical formulation to compute Lyapunov exponents and the main results. Finally, the main patterns deduced for the pluviometric regime are summarised and discussed in Sect. 5.

\subsection{Collection of data}

The dataset consists of daily pluviometric records covering the period 1950-1990 obtained from 43 rain gauges belonging to the Meteorological Services of the Spanish and Portuguese Governments. These rain gauges were carefully chosen on account of their excellent recording continuity and they have recently been used in statistical modelling of dry spell distribution (Lana et al., 2006). When a minor lack of data is detected, dry spells including days with missing data are discarded for computations. This decision does not strongly affect results concerning Hurst and Lyapunov exponents because the episodes of missing data are very scarce and event nonexistent for some rain gauges. When moving windows used to compute lacunarity include lack of data, they are also discarded for computations. Nevertheless, lacunarity should not be strongly affected by a few missing data, especially when a high number of moving windows are available. Figure 1 depicts the location of these rain gauges which are quite homogeneously distributed throughout the Iberian Peninsula. Five different series are gener-
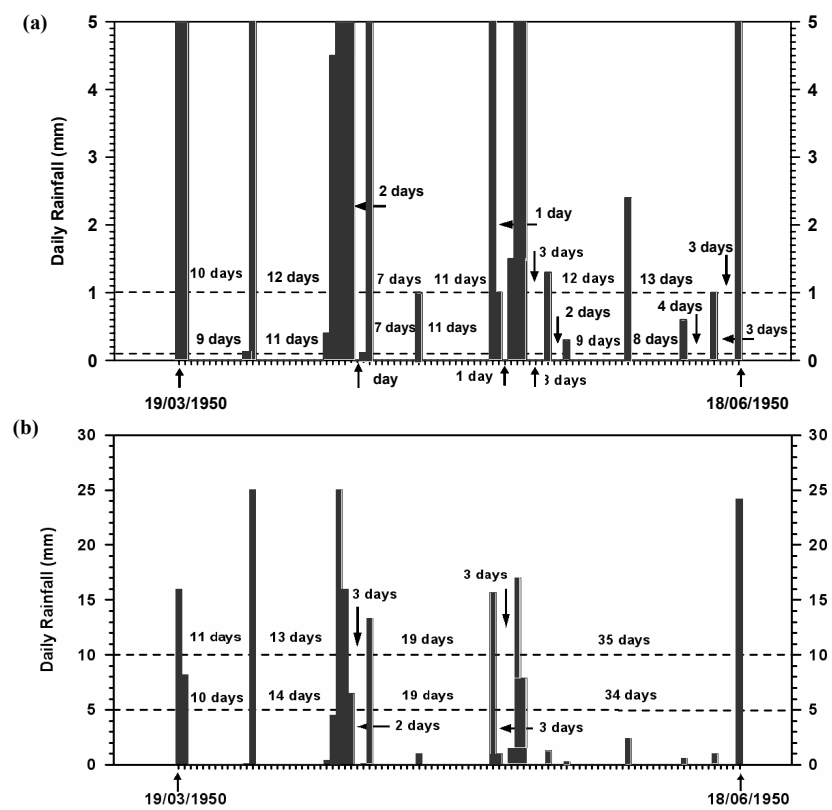

Fig. 2. Daily rainfall amounts recorded at rain gauge FAB (vertical bars) from 19 March to 18 June of 1950. Threshold levels of (a) 0.1 and $1.0 \mathrm{~mm} /$ day and (b) 5.0 and $10.0 \mathrm{~mm} /$ day are considered to illustrate the dry spell length sequence.

ated for every rain gauge. The first is the series of the daily rainfall amounts. The other four are formed by consecutive dry spell episodes. A dry spell is defined as the number of consecutive days with rainfall amounts lowering a threshold level. Four threshold levels $(0.1,1.0,5.0$ and $10.0 \mathrm{~mm} /$ day) are considered. The first level is the resolution of the pluviometers and it represents a simple distinction between "wet" and "dry" days. The other three levels distinguish between episodes from very moderate $(1.0 \mathrm{~mm} /$ day $)$ to remarkable daily amounts $(10.0 \mathrm{~mm} /$ day $)$. Figures $2 \mathrm{a}$ and $\mathrm{b}$ schematise the set of consecutive daily rainfall amounts for the rain gauge FAB (northern Mediterranean coast) during the period 19 March-18 June 1950 and the associated dry spells for the four different daily threshold levels. For instance, consecutive lengths of 11, 13, 3, 19, 3 and 35 days are detected within the recording period for a threshold level of $10.0 \mathrm{~mm} /$ day. As expected, Figs. $2 \mathrm{a}$ and $\mathrm{b}$ show that spell lengths tend to be fragmented with the decreasing threshold level.

\subsection{Methodology}

The pluviometric regime of the Iberian Peninsula is characterised by a remarkable spatial variability due to the great climatic differences among the inner Peninsula (Central Plateau) and the Atlantic and the Mediterranean influenced areas. Additionally, the orography, the complexity of the atmospheric circulation dynamics at mesoscale and, sometimes, the time irregularity of the Mediterranean climate 
also contribute to yield complex spatial and temporal patterns of the pluviometric regime. These patterns cannot be completely analysed and described by means of conventional tools such as statistical distributions, return periods or periodicities deduced from power spectra analyses (Lana et al., 2004, 2006; Burgueño et al., 2005; Martínez et al., 2007). Specifically, lacunarity, predictability of pluviometric records and instability of predictive algorithms are three aspects which cannot be properly analysed by those tools. The lacunarity of each daily pluviometric record is evaluated by using the concept and definition introduced by Mandelbrot (1982). The predictability (persistence, anti-persistence or randomness of a dataset) is qualified through the rescaled analysis and the meaning of the Hurst exponent (Feder, 1988; Korvin, 1992; Turcotte, 1997). The instability of possible predictive algorithms is quantified through the first two positive Lyapunov exponents (Eckmann et al., 1986; Stoop and Meier, 1988; Diks, 1999).

The lacunarity for each rain gauge is computed using moving windows of increasing length from 1 to 100 days, covering different time scales as days, weeks, months or seasons. Four different sets of lacunarity curves are obtained, one for each threshold level. In that way, the relevance of the lacunarity in the daily pluviometric records is evaluated by considering both the time period (window length) and the relevance of the episode (daily amount). It will be shown later that every lacunarity curve is very well described by a couple of power laws with exponents changing with the record (rain gauge) and depending on the threshold level. Additionally, lacunarity is expected to increase with the threshold level, on account of the behaviour of the spell lengths shown in Figs. 2a and $\mathrm{b}$.

The degree of persistence, anti-persistence and randomness of the five different sets of pluviometric data is deduced from the rescaled analysis, through the meaning of the Hurst exponent. Then, the predictability of both the daily pluviometric records and the dry spell length series can be checked. This analysis is especially relevant as it permits not only a better insight into the daily rain amount prevision, but a better knowledge of the behaviour of the dry spell lengths, which is very important when hazards concerning water resources and supplies have to be characterised.

Finally, the degree of instability of a predictive algorithm is a very important factor to delimit the necessary accuracy of the basic data (the pluviometer resolution for instance) and the possible success when predicting events. Since long series are needed to generate reconstructed vectors of the original data (Takens, 1981; Grassberger and Procaccia, 1983a, b) in order to accurately compute the Lyapunov exponents (Diks, 1999), only the series of daily rainfall and dry spell lengths for the threshold of $0.1 \mathrm{~mm} /$ day have been considered. Unfortunately, the number of consecutive dry spells for higher thresholds, especially for 5.0 and $10.0 \mathrm{~mm} /$ day, is not high enough to this purpose.

\section{Lacunarity}

The concept of lacunarity was introduced by Mandelbrot (1982) as a way of quantifying the distribution of gap sizes within a set of data. At the same time, it represents a measure of the failure of a fractal to be translationally invariant and also plays a relevant role in the study of critical phenomena. Moreover, several fractal sets characterised by the same fractal dimension can be distinguished by their lacunarities. For instance, it is possible to generate different Sierpinski carpet sets with the same fractal dimension, but with different lacunarity depending on the spatial concentration of gaps (Lin and Yang, 1986). Some illustrative examples of time series with different lacunarity can be found in Turcotte (1997). Whereas large lacunarity implies large gaps and clumping of points, small lacunarity suggests a rather uniform distribution with shorter gaps. Opposite examples could be synthetic series derived from a quite uniform distribution (low lacunarity) or a clumped distribution (high lacunarity). Intermediate examples could be those corresponding to a Cantor set or a random Cantor set. In this pluviometric analysis, the lacunarity is a measure of the distribution of segments, defined as "sequences of consecutive days" with rain amounts equalling to or exceeding a threshold amount, and gaps, defined as "sequences of consecutive days" with rain amounts below the selected threshold.

From a quantitative point of view, $n(s, r)$ is introduced as the number of moving windows of length $r$ (days) containing $\mathrm{s}$ segments (rainy days). After that, the probability $p(s, r)$ is evaluated as

$p(s, r)=n(s, r) / N(r)$

the total number of possible windows of length $r$ being

$N(r)=\ell-r+1$

with $\ell$ the total number of recording days, including segments and gaps. The first and second order moments of $s$ are

$$
\begin{aligned}
& M_{1}(r)=\sum_{s=1}^{r} s \cdot p(s, r) \\
& M_{2}(r)=\sum_{s=1}^{r} s^{2} \cdot p(s, r)
\end{aligned}
$$

and finally, the lacunarity is defined as the quotient

$$
L(r)=M_{2}(r) /\left[M_{1}(r)\right]^{2}
$$

It is worth mentioning that a different approximation to the evaluation of the lacunarity $L$ is given by the computation of the cluster dimension D (Korvin, 1992). An example applied to Earth sciences can be found, for instance, in Lana et al. (2005), who analysed elapsed times and distances between consecutive seismic events. Another example is the 

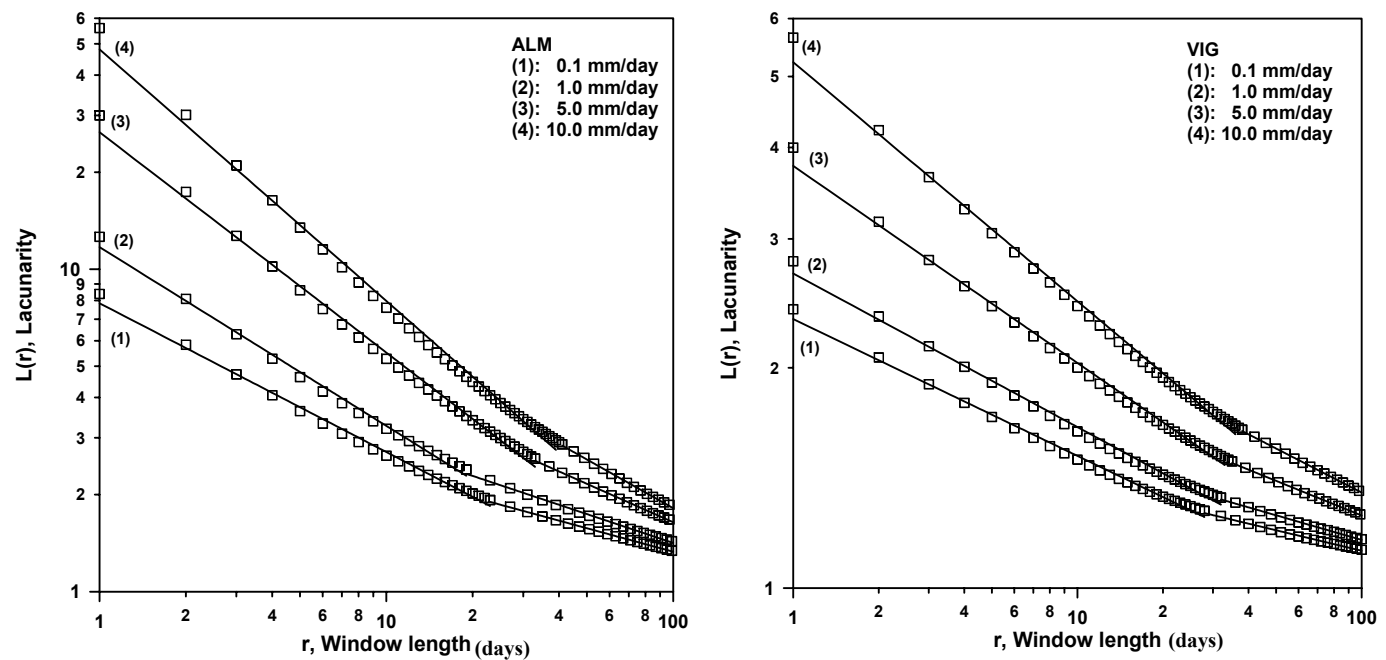

Fig. 3. Empiric lacunarity, L(r), (open squares) as a function of the window length $r$ for gauges ALM and VIG. Two power laws (solid lines) reproduce quite well the evolution of $\mathrm{L}(\mathrm{r})$, whatever the daily amount threshold.

study of Mazzarella (1999), who used the concepts of cluster dimension and Cantor dust to analyse the characteristics of the daily rainfall for two meteorological observatories in Italy. There are some similarities between the concepts of lacunarity $\mathrm{L}$ and cluster dimension $\mathrm{D}$. In rainfall processes, both methods try to quantify the time distribution of dry days or days with amounts lowering a threshold value (gaps). High values of $L$ imply long gaps. On the contrary, low values of $L$ suggest a quite uniform distribution of short gaps. From the viewpoint of the cluster dimension, the more isolated the clusters of rainy days (rainfall concentrated in a few consecutive days between long gaps), the smaller the value of D. Thus, small values of D would be related to high values of $L$. Conversely, low values of $L$ and high values of $\mathrm{D}$ would be associated with quite uniformly distributed short gaps.

The lacunarity of the pluviometric regime in the Iberian Peninsula is evaluated for different window lengths $r$ from 1 to 100 days at steps of one day. In this way, a detailed description of the lacunarity is achieved, especially at daily, weekly, monthly and seasonal scales, which are of especial interest in Climatology and Hydrology. Figure 3 depicts the lacunarity obtained for gauges ALM and VIG. Very similar curves are found for the rest of rain gauges. For all the threshold levels, the empirical lacunarities are quite well fitted by two power laws

$$
\begin{aligned}
& L(r)=\alpha \cdot r^{\beta} ; r=1, \ldots ., r_{c} \\
& L(r)=\delta \cdot r^{\varepsilon} ; r=r_{c}+1, \ldots . . r_{\max }
\end{aligned}
$$

with negative exponents $\beta$ and $\varepsilon$, always being $|\beta|>|\varepsilon| . \mathrm{r}_{c}$ represents the critical window length for which the power law changes from Eqs. (5a) to (5b), its value depending on the threshold level. An automated detection of the critical window length for every rain gauge and threshold level is questionable. $r_{c}$ is visually determined by testing the power laws (5a) and (5b) for different $r_{c}$ values. $r_{\max }$ is the maximum window length (100 days). A relevant feature is that, for a fixed window length, $L(r)$ always increases with the threshold level. The comparison of the two examples shown in Fig. 3 suggests that different pluviometric regimes are manifested by quite different lacunarities. Specifically, rain gauge VIG is submitted to an Atlantic regime, where drought periods are usually short (Lana et al., 2006) and annual rainfall amounts are very remarkable (annual average amount close to $1900 \mathrm{~mm}$ ). The counterexample is the rain gauge ALM, in the southern Mediterranean coast, where the drought periods can be very severe and the annual rainfall amounts very low (annual average amount close to $200 \mathrm{~mm}$ ). Effectively, an exhaustive revision of the results derived for the 43 rain gauges confirms that rain gauges submitted to Atlantic pluviometric regimes depict lower lacunarities than those corresponding to the Mediterranean regime (especially the southern Mediterranean) and the inner Peninsula (Central Plateau). Consequently, the lacunarity becomes a useful parameter to distinguish between different pluviometric regimes. Figure 4 depicts the spatial distribution of the lacunarity for window lengths of 1 and 30 days and threshold levels of $0.1,1.0,5.0$ and $10.0 \mathrm{~mm} /$ day. It is worth mentioning the strong reduction of the lacunarity from 1 to 30 days for all the threshold levels. In this set of maps and the forthcomings, the standard kriging interpolation (Oliver and Webster, 1990) has been used to obtain accurate spatial descriptions of the variables analysed.

Another interesting question is the spatial distribution of the exponents of the power laws given by Eqs. (5a) and (5b), especially the exponent $\beta$, which is associated with 

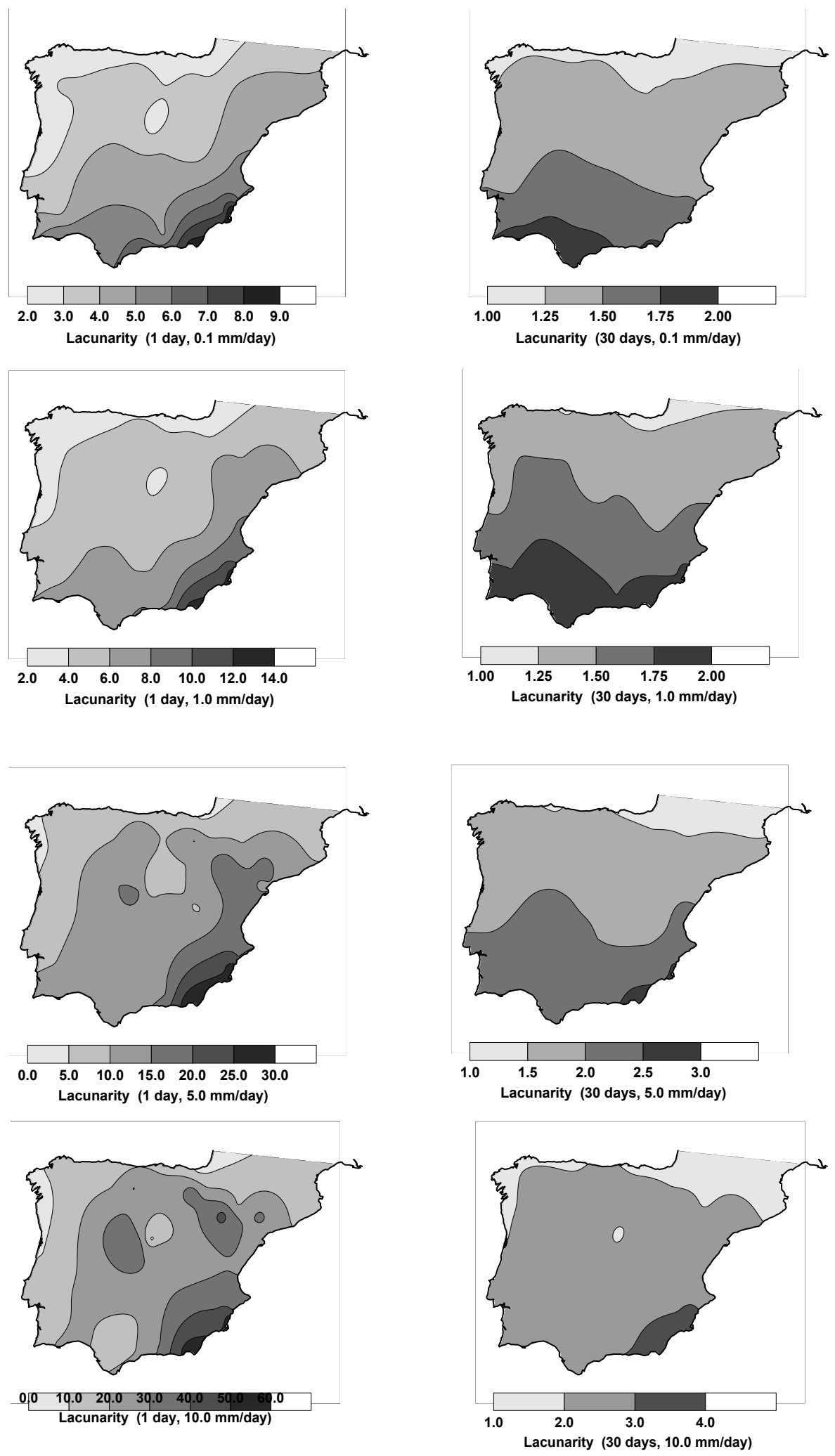

Fig. 4. Spatial distribution of the lacunarity for daily amount thresholds of $0.1,1.0,5.0$ and $10.0 \mathrm{~mm} /$ day and window lengths of 1 and 30 days. 

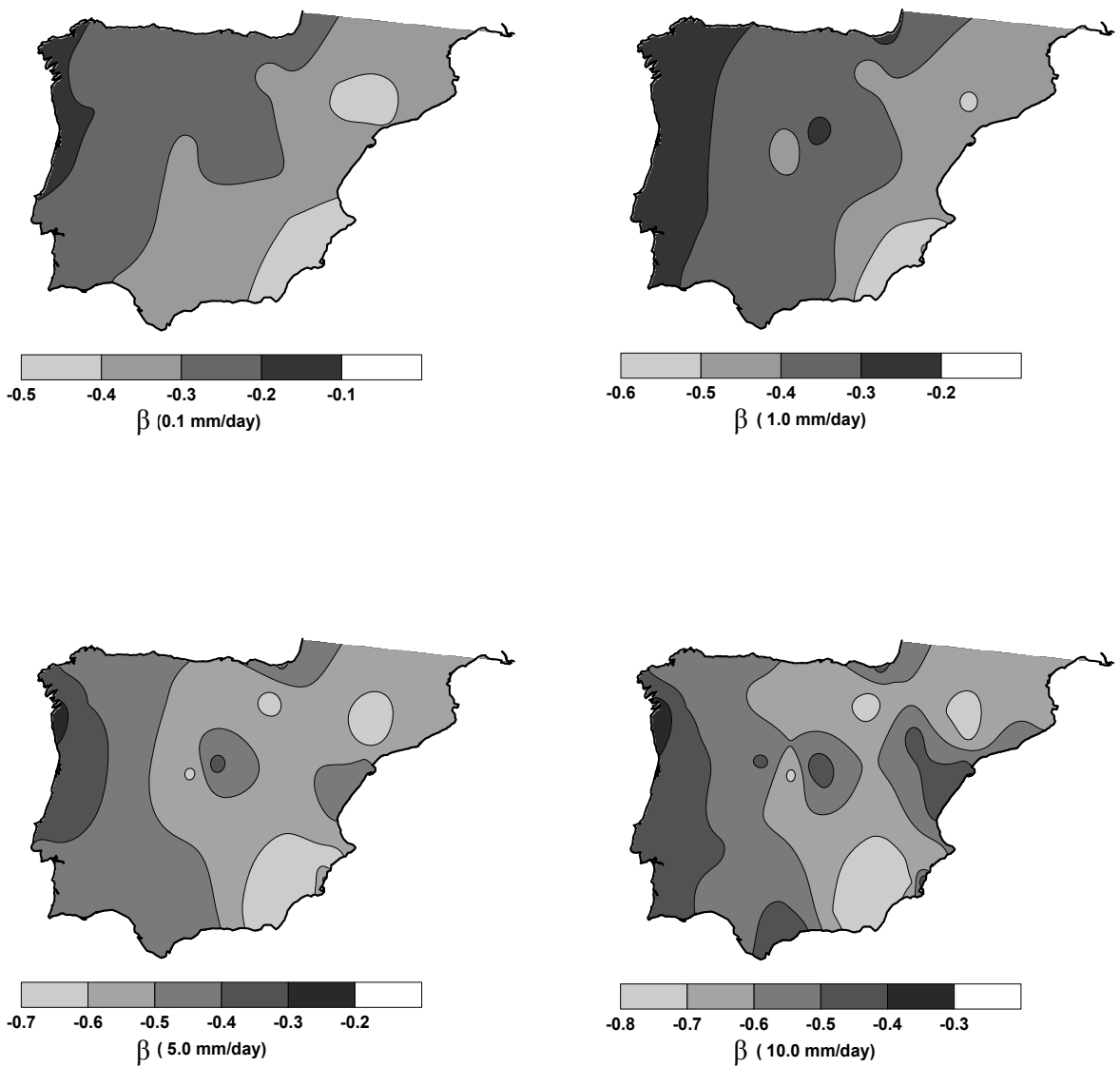

Fig. 5. Spatial distribution of the exponent $\beta$ of the power law reproducing lacunarity for window lengths ranging from 1 to $r_{c}$ (days) and daily amount thresholds of $0.1,1.0,5.0$ and $10.0 \mathrm{~mm} /$ day.

the greatest rate of reduction of the lacunarity with the window length. Figure 5 depicts the spatial distribution of $\beta$ for the four daily amount thresholds. As a general feature, for the level of $0.1 \mathrm{~mm} /$ day, the western part of the Iberian Peninsula shows the smallest reduction of the lacunarity with the increasing window length, whereas the greatest reduction is observed in eastern and, especially, in the south-eastern Spain. A quite similar pattern still remains for $1.0 \mathrm{~mm} /$ day, but not for greatest thresholds, especially, for $10.0 \mathrm{~mm} / \mathrm{day}$, though small values of $\beta$ are again detected in wide areas of south-eastern Spain. The second power law, with the exponent $\varepsilon$, describes the evolution of lacunarity close to 1.0 (the minimum asymptotic lacunarity) for window lengths $r$ that often are close to or exceed one month.

\section{Predictability}

The rescaled analysis and the Hurst exponent provide us with an interesting point of view of the behaviour of a complex dynamic system (Feder, 1988; Goltz, 1997). Applications to a variety of fields in Geology and Geophysics can be found, for instance, in Korvin (1992) and Turcotte (1997). Some examples of analyses of rainfall series for different spatial domains at various time scales can be found in Oñate (1997), Miranda and Andrade (1999, 2001), Whiting et al. (2003) and Miranda et al. (2004), among others. Very briefly, the rescaled process consists of the computation of mean values, cumulative differences, and after that, maximum range of the integrated signal, $R(\tau)$, and standard deviations, $S(\tau)$, for subsets of series with different number $\tau$ of consecutive elements. If there exists fractal behaviour, the Hurst exponent, $H$, defined as positive, is introduced through the expression

$$
R(\tau) / S(\tau)=a \tau^{H}
$$

Values of $H$ close to 0.5 suggest the randomness of the magnitude analysed. Then, successive steps of the complex dynamic system are independent and the best prediction is the last measure. Values of $H$ clearly exceeding 0.5 indicate time persistence of the dynamic system. It implies that the time trend deduced for the analysed set of length $\tau$ will remain and the best prediction must be based on the extrapolation of this trend. Finally, values of $H$ well below 0.5 suggest antipersistence. Thus, the time trend will reverse and the best prediction is the average over the length $\tau$. 
The predictability of the pluviometric regime of the Iberian Peninsula is analysed considering the five data series generated for each rain gauge as an accurate estimation of $H$ does not always need a very large number of data samples. For this purpose, it is required, first, a low uncertainty on $H$. Second, an acceptable square regression coefficient, derived from the representation of $\log \{\mathrm{R}(\tau) / \mathrm{S}(\tau)\}$ in terms of $\log \{\tau\}$, is also recommended. Finally, the linear evolution of the log$\log$ representation should cover two or more magnitude orders of $\tau$. A careful check on uncertainties on $H$ and square regression coefficients reveals that reliable Hurst exponents are obtained for the 43 rain gauges and the five pluviometric series. Uncertainties on $H$ are usually small and they affect the second decimal digit at the most. With respect to the range used for the rescaled analysis, $\tau$ arrives up to 4000, $1000,800,400$ and 200 elements for the consecutive daily rainfalls and the four dry spell series respectively. Given that values of $\tau$ less than 10 are not considered to avoid computational artefacts, results for thresholds of 5.0 and especially $10.0 \mathrm{~mm} /$ day should be carefully considered since two magnitude orders of $\tau$ are not achieved. Figure 6 shows the ratio $\mathrm{R} / \mathrm{S}$ for rain gauge ZAR. In this example, whereas the series of consecutive daily amounts and, especially, dry spells respect to $0.1 \mathrm{~mm} /$ day depict clear randomness (values of $H$ close to 0.5 ), the dry spells series for 1.0 and $10.0 \mathrm{~mm} /$ day show some signs of persistence (values of $H$ nearing 0.6) and dry spells for $5.0 \mathrm{~mm} /$ day should be governed by persistence $(H=0.61)$. Then, it should be more likely a successful prediction of dry spells related to a threshold level of $5.0 \mathrm{~mm} /$ day than for levels of 1.0 and $10.0 \mathrm{~mm} /$ day and even more than estimations of future daily rain amounts or dry spells for levels of $0.1,1.0$ and $10.0 \mathrm{~mm} /$ day. A shortcoming of the rescaled analysis of dry spells for high threshold levels is the relatively short number of elements of the series. Then, although the persistence detected for $5.0 \mathrm{~mm} /$ day, this result has to be carefully considered since the constraint of covering at least two cycles of the logarithmic scale of $\tau$ is not fully accomplished.

Figure 7 depicts the spatial distribution across the Iberian Peninsula of the Hurst exponent for the five series. Solid thick lines delimit areas governed by persistence and antipersistence $(H=0.5)$. Signs of anti-persistence are not detected for the daily rainfall series. A wide area of the Iberian Peninsula depicts signs of persistence $(0.5<H<0.6)$ and only a small domain in the south can be associated with a relevant persistence $(0.7<H<0.8)$. The spatial patterns for the dry spell series are quite different. The limit between areas governed by persistence and anti-persistence tend to drift from north to south as the threshold level increases. Nevertheless, a substantial reduction of the areas of antipersistence is only evident for 5.0 and $10.0 \mathrm{~mm} /$ day. Clear signs of persistence $(0.6<H<0.7)$ are only detected in some unconnected domains of the Iberian Peninsula for the threshold level of $5.0 \mathrm{~mm} /$ day.

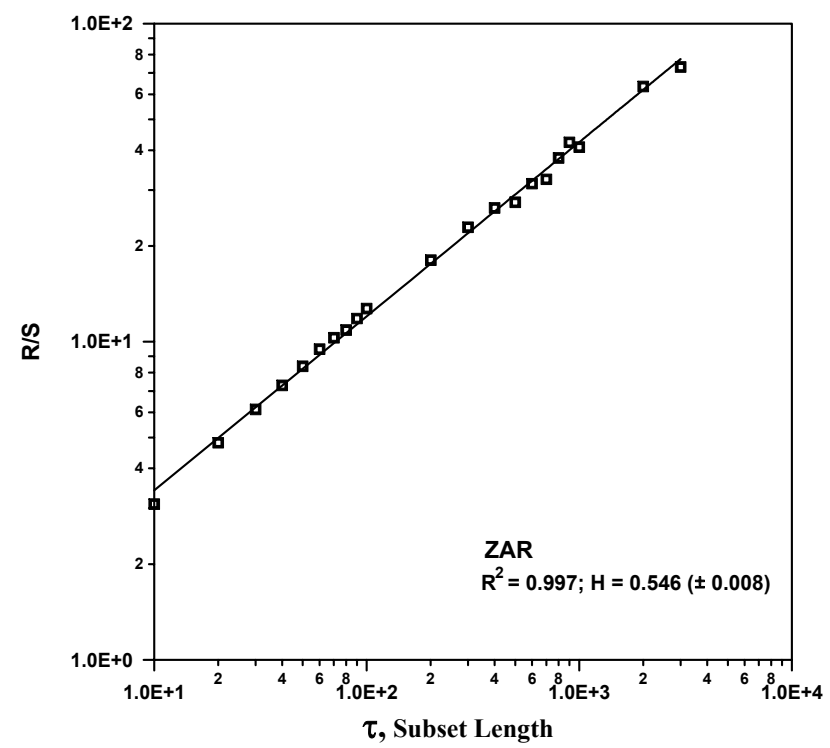

Fig. 6a. Ratio R/S for rain gauge ZAR based on the series of consecutive daily amounts.

\section{Predictive instability}

Uncertainties and instabilities concerning predictive algorithms applied to complex mechanisms such as the daily rainfall regime can be delimited through the Lyapunov exponents, which give a measure of how small uncertainties on the starting conditions of a complex system could lead to large errors in predictions of its future state. The Lyapunov exponents are introduced on the basis of the reconstruction theorem and reconstructed vectors (Takens, 1981; Grassberger and Procaccia, 1983a, b). The main objective is quantifying the predictive instability by computing the first two Lyapunov exponents. In addition, the reconstruction theorem permits a complete description of a dynamical complex system by means of the concepts of correlation dimension and Kolmogorov entropy (Diks, 1999).

The pluviometric datasets are generically represented by $\{x(k), k=1, \ldots, n\}$, with $\mathrm{n}$ the number of samples of each series and $x(k)$ either consecutive daily rainfalls or spell lengths. The space of our dynamical system is reconstructed by a set of $m$-dimensional vectors

$Z_{i}=\{x(i), x(i+1), \ldots, x(i+m-1)\} ; \quad i=1, \ldots, n-m+1(7)$

A very simple reconstruction of a dynamical system would be that obtained from dimension $m$ equal to 2 . It should be something similar to assume, for instance, that a daily rainfall amount depends solely on the previous amount. The behaviour of the dynamical system is, of course, much more complex and higher dimensions are necessary for a right description. A daily rainfall amount could depend, in a very complex way, on many previous rainfall episodes and the reconstructed space needs for vectors $Z_{i}$ generated with $\mathrm{m}$ 

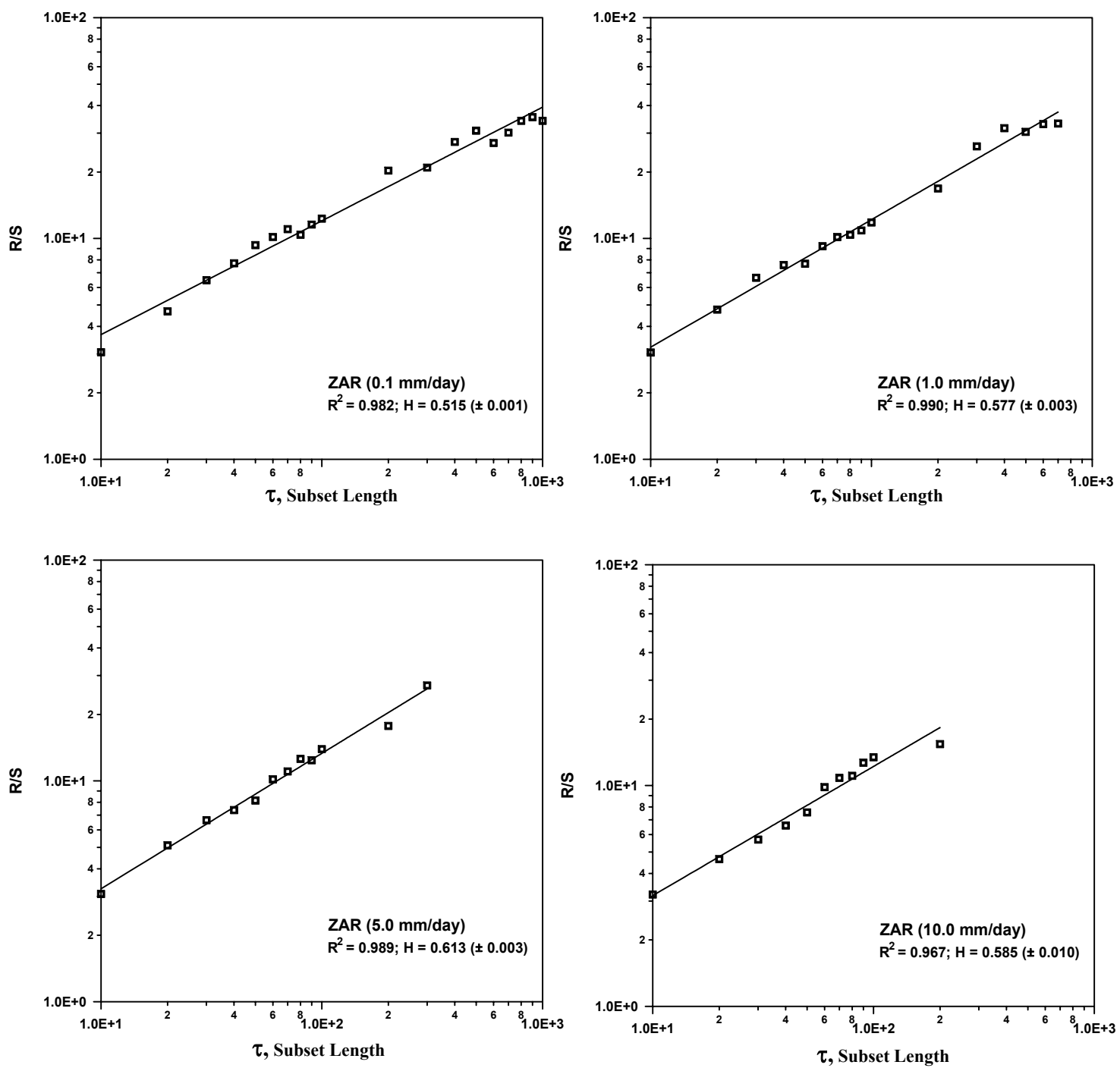

Fig. 6b. Ratio R/S for rain gauge ZAR based on the four series of dry spells generated for threshold levels of 0.1, 1.0, 5.0 and 10.0 mm/day.

higher than 2. Equation (7) generates a set of vectors, where (m-1) components of $Z_{i-1}$ are also used for $Z_{i}$, and the number of previous daily rainfall amounts or dry spell lengths used to explain the next episode increases with $\mathrm{m}$. An excessively high dimension $m$ would generate redundant or useless information. Thus, it is convenient to search for the optimal dimension $\mathrm{m}$, usually designed as embedding dimension $d_{E}$, for the best reconstruction of the space of the dynamical system. If the embedding dimension is estimated in some way, the largest Lyapunov exponent, responsible of the instability, is introduced as

$\lambda_{1}=\lim _{i \rightarrow \infty}\left\{\frac{1}{i} \ln \left\|\frac{\delta Z_{i}}{\delta Z_{0}}\right\|\right\}$

with $\delta Z_{0}$ being an infinitesimal change on the starting point in the reconstructed space of the dynamical system, $\delta Z_{i}$ the change on the reconstructed vector due to instability, after the $i$-th step in the reconstructed space, and $|\bullet|$ the norm of the Jacobian matrix. If $\lambda_{1}$ is positive, a future state of the dynamical system could strongly depend on uncertainties on the initial state. Then, the long-term prediction (large i) would be submitted to an outstanding instability.

Non-negative Lyapunov exponents can be numerically estimated from a time series according to the procedure proposed by Wolf et al. (1985). A more sophisticated computational algorithm (Eckmann et al., 1986; Stoop and Meier, 1988) permits to obtain all the positive and negative Lyapunov exponents, $\lambda_{i}\left(\mathrm{i}=1, \ldots, \mathrm{d}_{E}\right)$ and a good estimation of the embedding dimension. First, it is assumed that a function $\mathrm{f}(\bullet)$ permits to relate the different reconstructed vectors following the relationship

$$
Z_{k}=f\left(Z_{k-1}\right)=\ldots .=f^{k}\left(Z_{0}\right)
$$



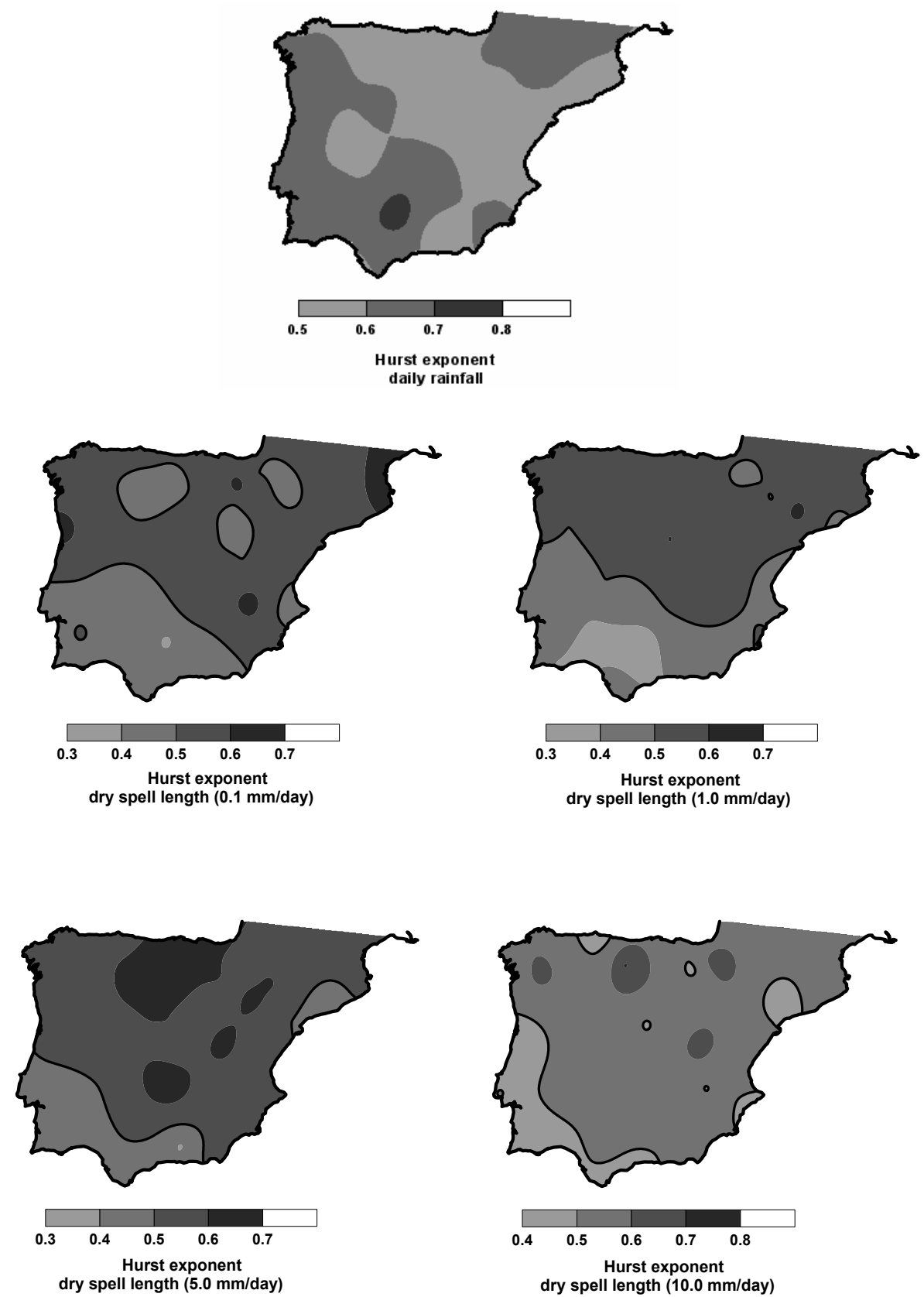

Fig. 7. Spatial distribution of the Hurst exponent for the series of daily rainfall and the series of dry spell lengths for the different threshold levels. Solid thick line delimits the areas related to persistence and anti-persistence $(H=0.5)$.

with $Z_{0}$ being the starting point in the reconstructed space or, in other words, the first reconstructed vector according to the recurrence given by Eq. (7). According to Eq. (8), the main objective is to quantify the instability of the dynamic system by considering small perturbations at the starting point. For this purpose, it can be considered the development of $f^{k}$ in terms of Taylor's series around $Z_{0}$

$\left\|f^{k}\left(Z_{0}\right)-f^{k}\left(Z_{0}+V_{0} \varepsilon\right)\right\|=\left\{\prod_{i=0}^{k-1} D f\left(Z_{i}\right)\right\} V_{0} \varepsilon+O\left(\varepsilon^{2}\right)$ with $V_{0}$ an arbitrary vector, $\varepsilon$ less than 1.0 and $\operatorname{DF}\left(Z_{i}\right)$ representing the partial derivative matrix of the function $f$. Thus, $V_{0} \varepsilon$ represents the arbitrary small perturbation at the starting point. The use of Eq. (10), with the arbitrary term $V_{0} \varepsilon$, and Eq. (8) to obtain infinitesimal changes on the starting point, $\delta Z_{0}$, and on the $i$-th step, $\delta Z_{i}$, is a valid option to determine the largest Lyapunov exponent. Nevertheless, all the exponents, including $\lambda_{1}$, can be estimated by taking into account they are the eigenvalues of the partial derivative matrix DF. 
In this way, the arbitrary term $V_{0} \varepsilon$ is not necessary. The matrix $\mathbf{D F}$ can be expressed as the product of matrices

$D f\left(Z_{i}\right)=A\left(Z_{i}\right) B^{-1}\left(Z_{i}\right)$

the elements of the matrices A and B defined as

$$
\begin{aligned}
& A\left(Z_{i}\right)_{\alpha, \beta}=\sum_{m=1}^{M} \Pi_{m \alpha}^{i+1} \Lambda_{m \beta}^{i} \\
& B\left(Z_{i}\right)_{\alpha, \beta}=\sum_{m=1}^{M} \Lambda_{m \alpha}^{i} \Lambda_{m \beta}^{i}
\end{aligned}
$$

with indices $\alpha$ and $\beta$ ranging from 1 to the embedding dimension $d_{E}$ and

$$
\begin{aligned}
& \Pi^{i+1}=f\left(Y_{i}\right)-f\left(Z_{i}\right) \\
& \Lambda^{i}=Y_{i}-Z_{i}
\end{aligned}
$$

$M$ is the number of samples of reconstructed vectors $Y_{i}$ around $Z_{i}$ and of samples $f\left(Y_{i}\right)$ around $f\left(Z_{i}\right)$ The optimal number of samples is the minimum of the pair $\left\{2 d_{E}, d_{E}+4\right\}$ according to Stoop and Meier (1988). After computing the partial derivative matrix, a base of orthonormal vectors $\left\{V_{1}^{0}, \ldots, V_{d_{E}}^{0}\right\}$ is required to start the following iterative computational process:

$W_{n}^{j}=D f\left(Z_{j-1}\right) V_{n}^{j-1} ; \quad n=1, \ldots, d_{E}$

$d_{1}^{j}=\left\|W_{1}^{j}\right\|, d_{m}^{j}=\left\|\omega_{m}^{j}\right\| ; \quad m=2, \ldots, d_{E}$

$\omega_{m}^{j}=W_{m}^{j}-\sum_{i=1}^{m-1}\left(V_{i}^{j}, W_{m}^{j}\right) V_{i}^{j}$

$V_{n}^{j}=\omega_{n}^{j} / d_{m}^{j}$

Equations (15) to (18) are repeated many times to obtain sets of $\left\{d_{1}^{j}, d_{2}^{j}, \ldots, d_{d_{E}}^{j}\right\}$ for $\mathrm{j}=1, \ldots, \mathrm{k}$, and finally the $n$-th eigenvector ( $n$-th Lyapunov exponent) is computed as

$\lambda_{n}=\lim _{k \rightarrow \infty} \frac{1}{k} \sum_{j=1}^{k} \log \left(d_{n}^{j}\right)$

An accurate computation of Lyapunov exponents according to Eq. (19) requires to control the evolution of each of them towards an asymptotic value for a large enough index $k$. In addition, it must be considered that, as the stationary correlation dimension of the complex system has not been estimated previously, the optimal embedding dimension $d_{E}$ is not known either (Lana et al., 2005). Then, all the computational steps, from Eqs. (11) to (19), must be repeated for increasing dimensions of the reconstructed space until detecting an optimal value that is assumed as the embedding dimension. A Lyapunov exponent should be then determined by searching for its stationary value after a long enough iteration of Eqs. (15) to (18), and a large enough reconstruction dimension.

The generation of the reconstruction vectors constraints the determination of accurate Lyapunov exponents to long sets of data, especially for large embedding dimensions. Consequently, the Lyapunov exponents are only computed for the series of daily rainfall and dry spell lengths generated for the threshold level of $0.1 \mathrm{~mm} /$ day, in order to ensure a large enough data sample.

Figure 8 shows the evolution of the first two positive Lyapunov exponents, $\lambda_{1}$ and $\lambda_{2}$, with the number of iterations of Eqs. (15) to (18) and with the increasing reconstruction dimension for the series of daily rainfall amounts recorded at rain gauge ZAR. One thousand iterations and an embedding dimension of 15 are necessary to achieve asymptotic values for the first, $\lambda_{1}$, and the second, $\lambda_{2}$, Lyapunov exponents. Very similar number of iterations and embedding dimensions are necessary for the rest of rain gauges. The spatial distribution of the first positive exponent $\lambda_{1}$ is shown in Fig. 9. For the daily rainfall series, a clear increase of the instability (higher Lyapunov exponents) from western to southern Iberian Peninsula and a narrow fringe of minimum Lyapunov values (low instability) on the Pyrenees (northern Iberian Peninsula) can be observed. The spatial patterns for the series of dry spell lengths $(0.1 \mathrm{~mm} /$ day $)$ are very different. Now a gradient is detected from NE Spain (low instability) to SW Spain (high instability). Consequently, uncertainties concerning prediction of daily amounts and dry spell lengths do not have, in general similar spatial characteristics.

\section{Conclusions}

Daily rainfall records of 43 rain gauges belonging to the Meteorological Offices of the Spanish and Portuguese Governments have permitted to analyse some aspects of the nonlinear behaviour of the pluviometric regime of the Iberian Peninsula. Specifically, the series of daily rainfall amounts and consecutive dry spell lengths have been analysed from the viewpoint of their lacunarity, predictability (re-scaled analysis and Hurst exponent) and predictive instability (Lyapunov exponents).

The decrease of the lacunarity with the increasing window length is well reproduced for all the rain gauges by two power laws, whatever the daily threshold level. The first power law is the most relevant as it reproduces the evolution of the lacunarity for window lengths often nearing one month. The first power law models the most notable decreasing of the lacunarity, while the second power law usually reproduces its evolution for values close to the asymptotic lacunarity of 1.0. The spatial distributions of the lacunarity and of the exponent $\beta$ of the first power law typically depict a notable organisation with south to north, and southeast to north-west gradients, thus suggesting some degree 


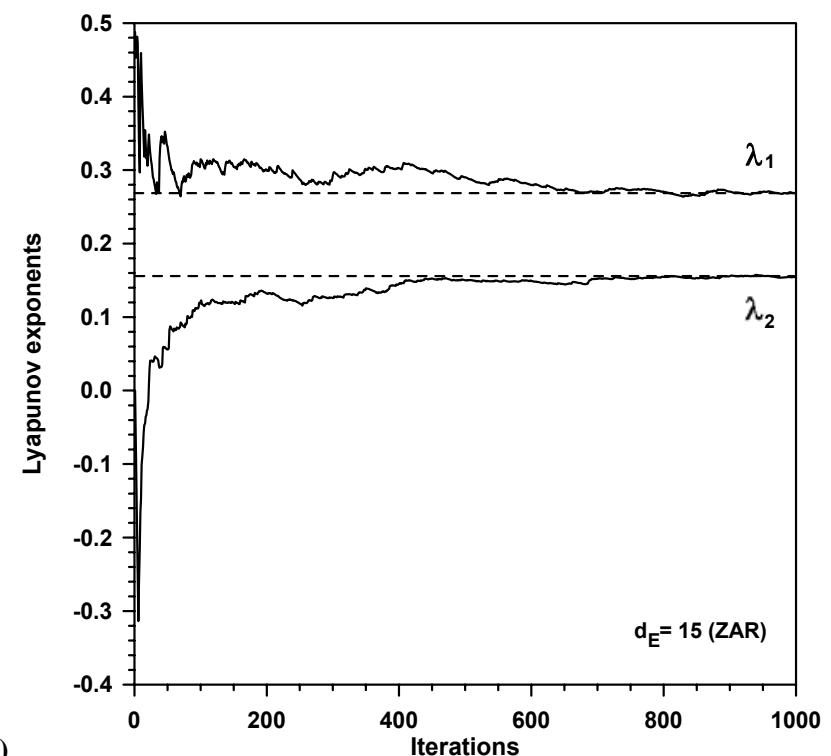

(a)

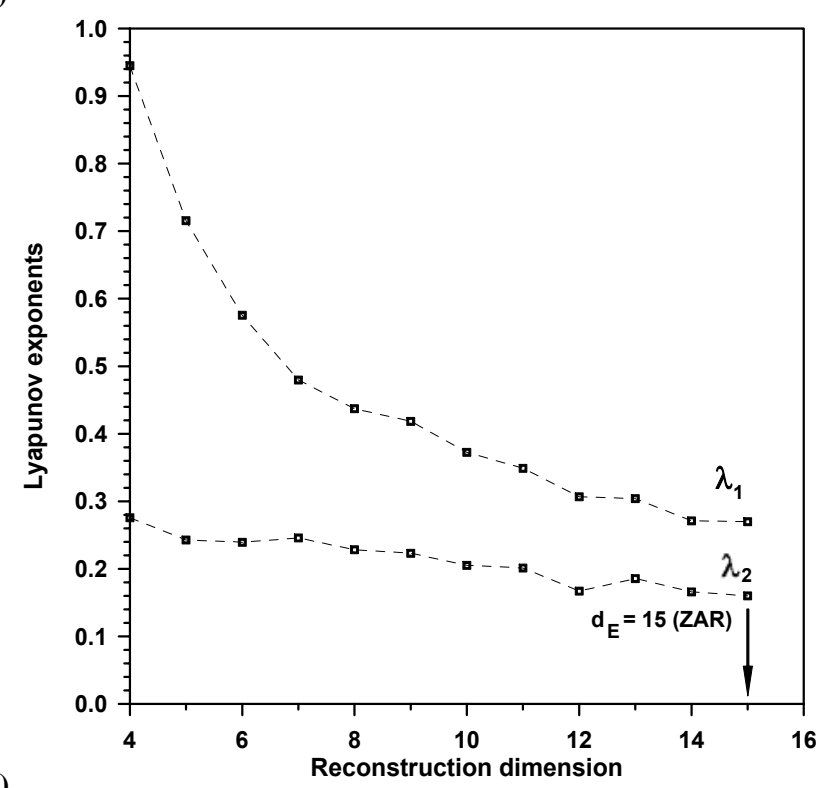

(b)

Fig. 8. (a) Evolution of the first two positive Lyapunov exponents with the number of iterations and (b) the reconstruction dimension for the series of daily rain amounts recorded at the rain gauge ZAR.

of Atlantic and Mediterranean climatic influences. It must be underlined, however, that patterns of the exponent $\beta$ for 5.0 and $10.0 \mathrm{~mm} /$ day threshold levels are less spatially organised.

The spatial distribution of the Hurst exponent is well organised for the five pluviometric series, but some differences are observed. Whereas the series of daily amounts presents either clear persistence or persistence close to randomness throughout the Iberian Peninsula, the series of dry spells mainly depict persistence to the north of the domain and antipersistence to the south. The randomness limit (isoline of

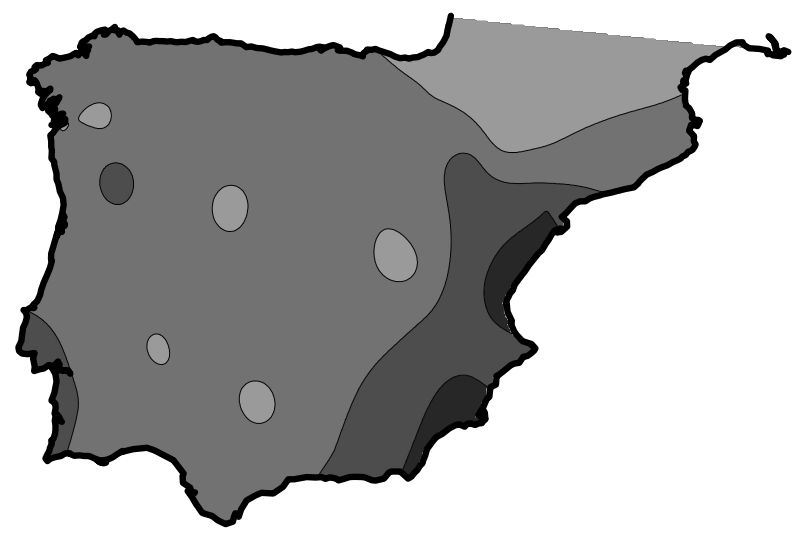

(a)
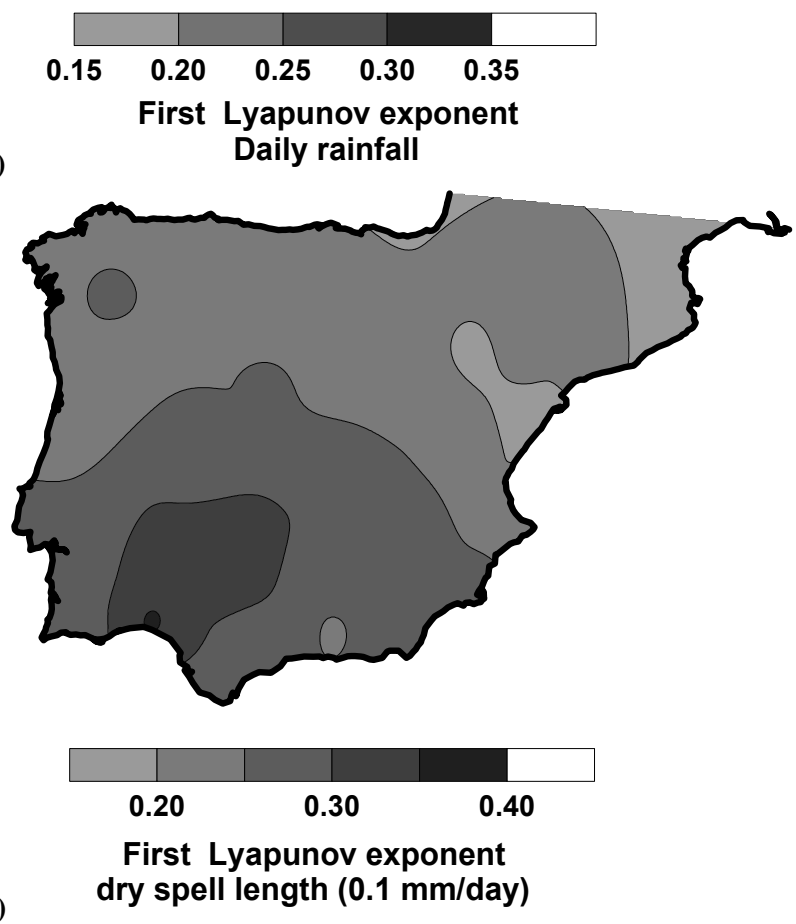

(b)

dry spell length $(0.1 \mathrm{~mm} /$ day $)$

Fig. 9. Spatial distribution of the first positive Lyapunov exponent for the series of (a) daily rainfall and (b) dry spell lengths $(0.1 \mathrm{~mm} /$ day) after one thousand iterations (embedding dimensions close to 15$)$.

$H=0.5$ ) shifts towards the south with the increasing threshold level. Consequently, a prediction of dry spell lengths based on extrapolation of previous time trends would not be reliable for the whole Iberian Peninsula. For some small southern areas, predictions should be based on the average of previous dry spell lengths.

With respect to the predictive instability, it must be kept in mind that it is strongly governed by the first positive Lyapunov exponent, $\lambda_{1}$. Consequently, the spatial distributions of $\lambda_{1}$ for daily rainfall and dry spell length $(0.1 \mathrm{~mm} /$ day $)$ series (Fig. 9) provide a good approximation to the predictive instability of the daily rainfall regime in the Iberian 
Peninsula. A common feature of all the series is the high embedding dimension, $d_{E}$, for which the first Lyapunov exponent achieves a stationary value. Dimension $d_{E}$ often equals or exceeds a value of 15 , which is a sign of a remarkable randomness component in the time behaviour of the daily amount and dry spell series. In addition to future analyses of correlation dimension and Kolmogorov entropy, which should give us some insight about the minimum number of non-linear equations governing the pluviometric regime and the "loss" of memory of the mechanism governing this complex process, the high embedding dimensions already enhance the complexity of the pluviometric regime. The spatial distribution of $\lambda_{1}$ is well organised, being especially relevant the clear north-west to south-west gradient for dry spell lengths. It is also worthy of mention that places where the predictability of the daily rainfall is very questionable (high Lyapunov exponent) do not match with those where instability affects the dry spell length prediction. The single exception is a narrow fringe in the southern Atlantic coast of Portugal, where both daily rainfall and dry spell length prediction should be submitted to relatively high uncertainties. Additionally, it must be mentioned that places in the Iberian Peninsula where the annual rainfall amounts are not very large (southern Spain), and drought periods are quite common, correspond to areas where prediction of dry spell lengths contributing to relevant droughts is submitted to significant uncertainty and instability.

As a summary, the present study introduces the first elements for a better knowledge of the daily rainfall regime of the Iberian Peninsula from the point of view of its nonlinear behaviour. Future studies intended for a deeper knowledge of the nonlinear behaviour of the daily pluviometric regime should also be based on the reconstruction theorem, but addressed to the evaluation of the correlation dimension and the Kolmogorov entropy.

Edited by: O. Talagrand

Reviewed by: A. Mazzarella and another referee

\section{References}

Burgueño, A., Martínez, M. D., Lana, X., and Serra, C.: Statistical distributions of the daily rainfall regime in Catalonia (NE Spain) for the years 1950-2000, Int. J. Climatol., 25, 1381-1403, 2005.

Diks, C.: Nonlinear Time Series Analysis. Methods and Applications. Nonlinear Time Series and Chaos, vol. 4., World Scientific, London, 1999.

Eckmann, J. P., Oliffson, S., Ruelle, D., and Ciliberto, S.: Lyapunov exponents from time series, Phys. Rew. (A), 34(6), 4971-4979, 1986.

Fedder, J.: Fractals, Ed. Plenum, New York, USA, 1988.

Goltz, C.: Fractal and Chaotic Properties of Earthquakes, in: Lecture Notes in Earth Sciences 77, Springer, Berlin, 1997.

Grassberger, P. and Procaccia, I.: Characterization of strange attractors, Phys. Rev. Lett., 50, 346-349, 1983a.
Grassberger, P. and Procaccia, I.: Estimation of the Kolmogorov entropy from a chaotic signal, Phys. Rev. (A), 28, 2591-2593, 1983b.

Harris, D., Menabde, M., Seed, A., and Austin, G.: Multifractal characterization of rain fields with a strong orographic influence, J. Geophys. Res., 101, 26 405-26414, 1996.

Hubert, P., Tessier, Y., Lovejoy, S., Shertzer, D., Schmitt, F., Ladoy, P., Carbonnel, J. P., Violette, S., and Desurosne, I.: Multifractals and extreme rainfall events, Geophys. Res. Lett., 20(10), 931934, 1993.

Korvin, G.: Fractal Models in Earth Sciences, Elsevier, Amsterdam, 1992.

Lana, X., Martínez, M. D., Serra, C., and Burgueño, A.: Spatial and temporal variability of the daily rainfall regime for Catalonia (NE Spain), 1950-2000, Int. J. Climatol., 24, 613-641, 2004.

Lana, X., Martínez, M. D., Posadas, A. M., and Canas, A.: Fractal behaviour of the seismicity in the Southern Iberian Peninsula, Nonlin. Processes Geophys., 12, 353-361, 2005, http://www.nonlin-processes-geophys.net/12/353/2005/.

Lana, X., Martínez, M. D., Burgueño, A., Serra, C., Martín-Vide, J., and Gómez, L.: Distribution of long dry spells in the Iberian Peninsula, years 1951-1990, Int. J. Climatol., 26, 1999-2021, 2006.

Lima, M. I. P. and Grasman, J.: Multifractal analysis of 15-min and daily rainfall from a semi-arid region in Portugal, J. Hydrol., 220, 1-11, 1999.

Lin, B. and Yang, Z. R.: A suggested lacunarity expression for Sierpinski carpets, J. Phys. A, Math. Gen., 19, L49-L52, 1986.

Lovejoy, S. and Mandelbrot, B. B.: Fractal properties of rain and a fractal model, Tellus, 37A, 209-232, 1985.

Mandelbrot, B. B.: The Fractal Geometry of Nature, Freemann, San Francisco, USA, 1982.

Martínez, M. D., Lana, X., Burgueño, A., and Serra, C.: Spatial and temporal daily rainfall regime in Catalonia (NE Spain) derived from four precipitation indices, years 1950-2000, Int. J. Climatol., 27, 123-138, 2007.

Mazzarella, A.: Multifractal dynamic rainfall processes in Italy, Theor. Appl. Climatol., 63, 73-78, 1999.

Mazzarella, A. and Tranfaglia, G.: Fractal characterisation of geophysical measuring networks and its implication for an optimal location of additional stations: an application to a rain-gauge network, Theor. Appl. Climatol., 65, 157-163, 2000.

Miranda, J. G. V. and Andrade, R. F. S.: Rescaled range analysis of pluviometric records in Northeast Brazil, Theor. Appl. Climatol., 63, 79-88, 1999.

Miranda, J. G. V. and Andrade, R. F. S.: R/S analysis of pluviometric records: comparison with numerical experiments, Physica A, 295, 38-41, 2001.

Miranda, J. G. V., Andrade, R. F. S., da Silva, A. B., Ferreira, C. S., González, A. P., and Carrera López, J. L.: Temporal and spatial persistence in rainfall records from Northeast Brazil and Galicia (Spain), Theor. Appl. Climatol., 77, 113-121, 2004.

Oliver, M. A. and Webster, R.: Kriging: a method of interpolation for geographical information system, Int. J. Geographical Information Systems, 4(3), 313-332, 1990.

Olsson, J., Niemczynowicz, J., and Berndtsson, R.: Fractal analysis of high-resolution rainfall time series, J. Geophys. Res., 98, 23 265-23 274, 1993.

Oñate, J. J.: Fractal analysis of climatic data: annual precipitation 
records in Spain, Theor. Appl. Climatol., 56, 83-87, 1997.

Rodríguez-Iturbe, I., Febres de Power, B., Sharifi, M. B., and Georgakakos, K. P. (1989). Chaos in rainfall, Water Resour. Res., 25, 1667-1675, 1989.

Salas, J. D., Kim, S. H., Eykholt, R., Burlando, P., and Green, T. R.: Aggregation and sampling in deterministic chaos: implication for chaos identification in hydrological processes, Nonlin. Processes Geophys., 12, 557-567, 2005, http://www.nonlin-processes-geophys.net/12/557/2005/.

Sivakumar, B.: Rainfall dynamics at different temporal scales: A chaotic perspective, Hydrol. Earth Syst. Sci., 5, 645-651, 2001a.

Sivakumar, B.: Is a chaotic multi-fractal approach for rainfall possible?, Hydrol. Processes, 15, 943-955, 2001b.

Sivakumar, B., Sorooshian, S., Gupta, H. V., and Gao, X.: A chaotic approach to rainfall disaggregation, Water Resour. Res., 37, 61$72,2001$.

Stoop, R. and Meier, P. F.: Evaluation of Lyapunov exponents and scaling functions from time series, J. Opt. Soc. Am. (B), 5, 1037 1045,1988

Svensson, C., Olsson, J., and Berndtsson, R.: Multifractal properties of daily rainfall in two different climates, Water Resour. Res., 32, 2463-2472, 1996.
Takens, F.: Detecting Strange Attractors in Turbulence, in: Lecture Note in Mathematics, edited by: Rand, D. A. and Young, L. S., Springer, Berlin, 1981.

Tessier, Y., Lovejoy, S., Hubert, P., Shertzer, D., and Pecknold, S.: Multifractal analysis and modelling of rainfall and river flows and scaling, causal transfer functions, J. Geophys. Res., 101, 26427-26 440, 1996.

Turcotte, D. L.: Fractal and Chaos in Geology and Geophysics (2nd edition), Cambridge University Press, 1997.

Veneziano, D., Bras, R. L., and Niemann, J. D.: Nonlinearity and self-similarity of rainfall in time and a stochastic model, J. Geophys. Res., 101, 26371-26392, 1996.

Whiting, J. P., Lambert, M. F., and Metcalfe, A. V.: Modelling persistence in annual Australian point rainfall, Hydrol. Earth Syst. Sci., 7, 197-211, 2003, http://www.hydrol-earth-syst-sci.net/7/197/2003/.

Wolf, A., Swift, J. B., Swinney, H. L., and Vastano, J. A.: Determining Lyapunov exponents from a time series, Physica, 16D, 285-317, 1985. 